\title{
Cavernous Hemangioma Occurred Between the Trapezius and Splenius Capitis Muscle
}

\author{
Woo Hyung Choi ${ }^{1}$, Chung Jae Lee ${ }^{1}$, Sung Han Oh${ }^{1}$, Bong Sub Chung ${ }^{1}$, \\ Jong Kook Rhim ${ }^{1}$, Kye Won Kwon ${ }^{2}$ \\ ${ }^{l}$ Department of Neurosurgery, of ${ }^{2}$ Pathology, Bundang Jesaeng General Hospital, Sungnam, Gyeonggi, Korea
}

Hemangiomas are the most common benign tumor of soft tissue. They are frequently seen on the trunk and extremities. In addition, most of them exist at the skin and subcutaneous layer, but fewer than $1 \%$ does in the intramuscular layer. For the diagnostic images of the intramuscular cavernous hemangioma, ultrasound (US), computed tomography (CT) and magnetic resonance imaging (MRI) are used currently. Multiple therapeutic methods are used, but surgical excision is considered as the most ideal treatment. We describe the recurred cavernous hemangioma occurred between the trapezius and splenius capitis muscle. The mass was well demarcated but scattered and infiltrated into the adjacent muscle layer, therefore, extensive resection was unavoidable. When determining a treatment regime for the hemangioma, it is essential to consider the size of the mass, cosmetic and functional aspects of the patient.

Key Words: Cavernous hemangioma $\cdot$ Trapezius and Splenius capitis $\cdot$ Cavernoma

\section{INTRODUCTION}

Hemangiomas are the most common benign tumor accounting for $7 \%$ of all soft tissue tumors. Although the etiology is unclear, it is possibly considered as congenital in origin. Histologically it is thought to be as harmatoma or neoplasm ${ }^{2,17)}$. Generally, hemangiomas are more commonly affected female than male. They are frequently seen on the trunk and extremities, but up to $20 \%$ of hemangiomas are located at the head and neck region ${ }^{2,13,15)}$. Although the majority are cutaneous or mucosal hemangioma, the intramuscular hemangiomas are extremely rare accounting for $0.8 \%$ of all hemangiomas ${ }^{25,19)}$. Because of the rarity of these tumors in the muscular layer, preoperative diagnosis has been made fewer than $8 \%$ of cases ${ }^{12)}$. A palpable mass is the most common initial presentation, and bruits, thrills, and pulsation are infrequent because of surrounding muscular fibrosis that hides the vascular nature of the tumor. Therefore, imaging methods such as Ultrasound (US),

- Received: April 24, 2012 - Revised: June 5, 2012

- Accepted: June 25, 2012

Corresponding Author: Chung Jae Lee, MD

Department of Neurosurgery, Bundang Jesaeng General Hospital,

255-2, Sungnam City, Gyeonggi Do, Korea,

Tel: +82-31-779-0282, Fax: +82-31-779-0895,

E-mail: pplane2@dmc.or.kr computed tomography (CT) and magnetic resonance imaging (MRI) are essential for the proper diagnosis. Pathologic confirmation is mandatory. This report describes a rare case of the intramuscular cavernous hemangioma involving the posterior cervical muscle layer and we reviewed the literature to inform the diagnostic and treatment options.

\section{CASE REPORT}

A 61-year-old male patient was admitted to our hospital complaining of painful posterior neck mass. Five years ago, he had already received a surgery for that mass at a regional hospital. At that time, the pathology confirmed the cavernous hemangioma. However, the mass has slowly regrown at the same region for the five years. His neck motions were limited. An enhanced CT scan showed approximately $5 \times 3 \times 5 \mathrm{~cm}$ sized well margined and multi-nodular calcified mass in the posterior neck muscle layer. The density of mass was similar to the surrounding muscles (Fig. 1). The mass was iso- to high signal intensity with low signal calcified lesion on the T1-weighted and high- to intermediated signal intensity with fluid-fluid level on the T2-weighted images. A T1-weighted gadolinium enhanced sagittal MRI revealed heterogeneous signal intensity between the trapezius and splenius capitis muscle and further enhanced as time passed (Fig. 2). Surgery was performed using a horizontal skin incision and the mass was removed comple- 


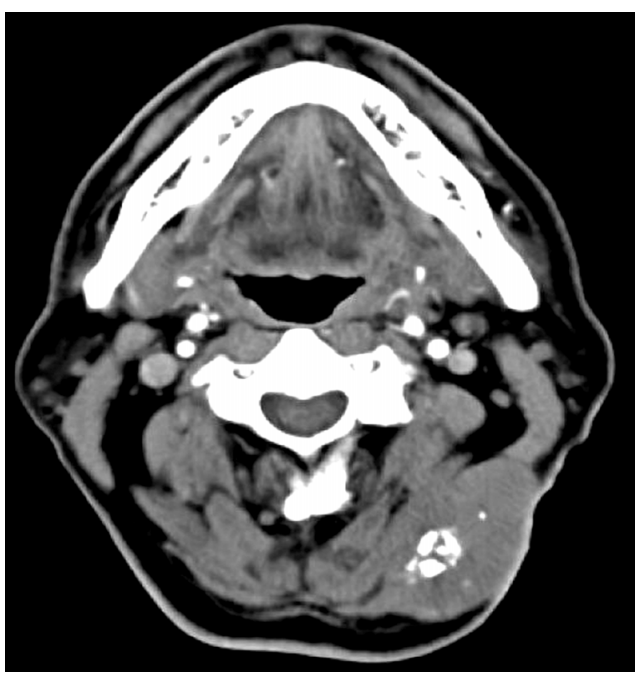

Fig. 1. An enhanced CT scan showing well margined and multinodular calcified mass in the posterior neck muscle layer.

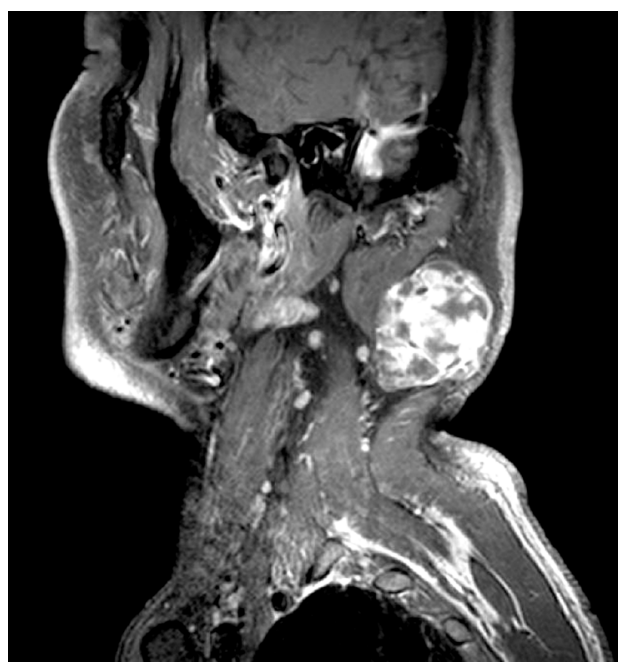

Fig. 2. A T1-weighted gadolinium enhanced sagittal MRI scan demonstrating heterogeneous signal intensity between the trapezius and splenius capitis muscle layer.

tely. Grossly the mass contained many vascular channels with focal bone formation (Fig. 3). Pathological result was cavernous hemangioma (Fig. 4). Postoperatively, his neck motions such as shoulder shrugging, neck extension, lateral flexion and rotation were normal. At the moment of a year passing, there is no evidence of recurrence.

\section{DISCUSSION}

Histologically, hemangiomas are classified based on the predominant type of vascular channel such as capillary hemangio-

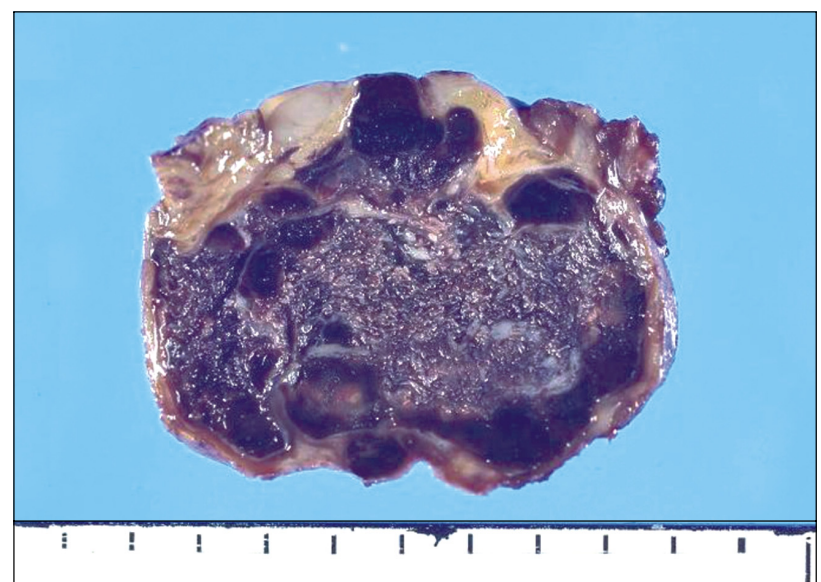

Fig. 3. A soft dark red mass revealing many dilated vessels of various caliber with focal bone formation.

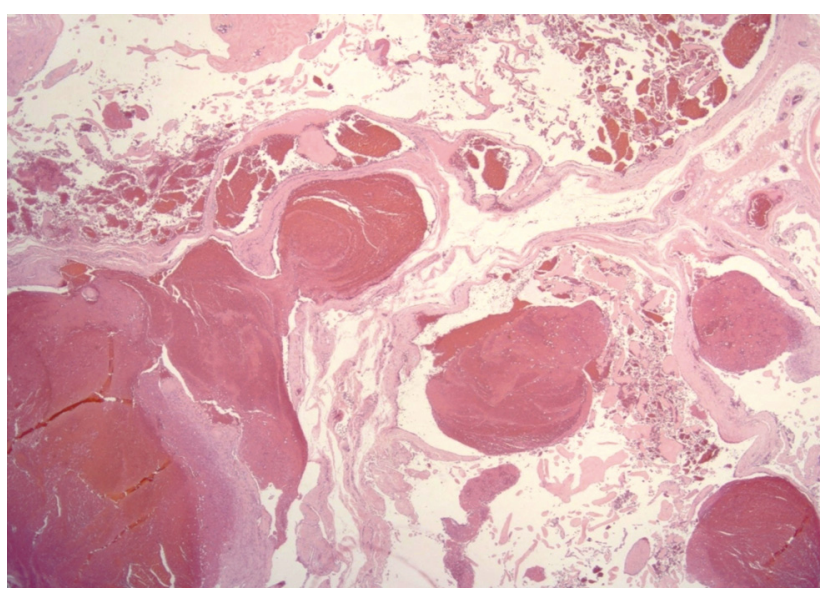

Fig. 4. Histological findings showing the mass composed of large, dilated, blood filled cavernous vascular spaces lined by flattened endothelim with partly numerous intraluminal papillae formation $(\mathrm{H} / \mathrm{E}, \times 40)$.

ma, cavernous hemangioma, arteriovenous hemangioma and venous hemangioma ${ }^{9}$. Cavernous hemangioma consists of large numbers of thin walled vascular channels lined by a single layer of endothelial cells and separated by fibrous connective tissue, whereas capillary hemangioma has no fibrous septa and smaller vascular lumens, some of which are filled with blood ${ }^{12)}$. The cavernous hemanioma is the most common type of the intramuscular hemangiomas ${ }^{10)}$. This intramuscular hemangioma is non-metastatic congenital tumor, and approximately fifty percents of them remains undetected until the other symptoms revealed. The most common symptom is pain mainly caused by mass effect ${ }^{2}$. Because of uncertainty in clinical diagnosis, various diagnostic methods such as US, CT, MRI and arteriography are used. Among them, CT and MRI scans are commonly used. A non-enhanced CT scan alone is usually ineffe- 
ctive in demonstrating these lesions because the density of the cavernous hemangioma approaches that of the surrounding muscle and soft tissues. The CT scan with contrast enhancement should demonstrate a vascular tumor, with the added advantage of ruling out the possibilities of bony erosion, major vessel involvement. An MRI scan has been shown to provide better detection and delineation of the extent of intramuscular cavernous hemangioma than CT scan. The coronal and sagittal images are extremely useful in assessing the relationships among the cavernous hemangioma, muscle, and other tissues. On T1-weighted images, cavernous hemangioma appears as a low-to-intermediate signal intensity mass with peripheral high signal intensity of the fat overgrowth. On T2-weighted images, it shows areas of high signal intensity of the vascular tissue and intermediate signal intensity of the fat. Characteristic tubular or serpentine components are orientated along the muscular long axis. Thrombi are hyperintense on T1-weighted and hypointense on T2-weighted sequences ${ }^{1,414,18)}$. An angiography should be considered if the MRI scan has not provided sufficient information about the relationship between the tumor and a neurovascular bundle. Although the cutaneous hemangiomas often regress during early childhood, spontaneous regression of the intramuscular hemangiomas has not been described ${ }^{3)}$. The treatment methods such as surgical excision, systemic steroid administration, intralesional steroid injection, sclerothrapy, cryotherapy, vascular ligation and embolization have been used $^{1,6,11,18)}$.

Complete surgical excision is essential for the definite treatment. Bella and Cheng et $\mathrm{al}^{7}$ ) reported that surgical margins and tumor size were the independent factors predicting local recurrence free survival. Incomplete surgical resection also leads increasing rate of recurrence. This relapse is due to its microscopically infiltrative pattern of diffusion into the surrounding muscular tissue, and minor arterial feeder vessels may also be responsible for recurrence of the hemangioma ${ }^{8)}$. Wolf et al. ${ }^{20)}$ and Tang et al. ${ }^{17)}$ reported local relapse rates of $18 \%$ and 19 $\%$ each other after total surgical intervention. In case of scattered hemangiomas that invade the adjacent muscles, exten sive resection is often unavoidable. This occasionally causes loss of function of the surrounding tissue. Therefore, multiple therapeutic methods should be considered depending on the state of the intramuscular hemangiomas.

We report a very rare case of the intramuscular cavernous hemangioma involving the posterior cervical muscle layer. The preoperative MRI was compatible with the cavernous hemagioma and pathologic findings confirmed the cavernous hemagioma. The patient had progressively complained of painful neck lump and limitation of neck motion after the first surgery. We presume that the local recurrence was from the previous incomplete resection.

\section{CONCLUSION}

Cavernous hemangioma has characteristic MRI and pathologic findings. In case of invasive intramuscular hemangioma or massive mass effect, adjuvant therapy and wide excision to prevent local recurrence should be considered ${ }^{1,6,11,18)}$. We report a rare case of surgically managed recurred cavernous hemangioma between the trapezius and splenius capitis muscle. For the intramuscular cavernous hemangioma, the complete surgical excision is essential for the prevention of recurrence. However, we should consider another therapeutic option for the cosmetic and functional aspects depending of the tumor size and location.

\section{REFERENCES}

1. Afsar FS, Oziz E, Hamdioglu Y, Karasoy I, Uguz B: Intramuscular hemangioma of the masseter muscle in 9-year old girl. Acta Angiol 13:42-46, 2007

2. Allan PW, Enzinger FM: Haemangioma of skeletal muscle, ananalysis of 89 cases. Cancer 29:8-22, 1972

3. Avci G, Yim S, Misirlioglu A, Akoz T, Kartal LK: Intramasseteric hemangioma: Plast Reconstr Surg 109:1748-50, 2002

4. Boricic I, Stojsic Z, Mikic A, Brasanac D, Tomanovic N, Bacetic D: Intramuscular hemangioma of the retropharyngeal space. Vojnosanit Pregl 64:485-488, 2007

5. Demir, Zuhtu; Oktem, Fatih; Celebioglu, Selim: Rare case of intramasseteric cavernous hemangioma in a three-year-old boy: a diagnostic dilemma. Ann Otol Rhinol Laryngol 113:455-458, 2004

6. Enneking WF, Campanacci M: Bone and Soft Tissue Tumors. 2nd ed. New-york, Springer-Verlag, 1051-1081, 2000

7. Bella GP, Manivel JC, Thompson RC Jr, Clohisy DR, Cheng ET: Intramuscular hemangioma; recurrence risk related to surgical margins. Clin Orthop Relat Res 459:186-191, 2007

8. Ingalls GK, Bonnington GJ, Sisk AL: Intramuscular hemangioma of the mentalis muscle. Oral Surg Oral Med Oral Pathol 60:476-481, 1985

9. Hein KD, Mulliken JB, Kozakewich HP, Upton J, Burrows PE: Venous malformations of skeletal muscle. Plast Reconstr Surg 110:1625-1635, 2002

10. Huh MC, Yoo CI and Lee JY: A clinical study on the cavernous hemangiomas developed in skeletal muscles of the extremities. J Korean Orthop Assoc 14:385-393, 1979

11. Jenkins HP, Delaney PA: Benign Angiomatous Tumors of Skeletal Muscles. Surg, Gynec, and Obstet 55:464-480, 1932

12. Jong-Hyuk Park, Suk-Hyung Kang, Seung Won Park: Spinal Epidural Cavernous Hemangioma Resembling a Metastatic Tumor in an 89-year-old Man. Korean J Spine 7(2):96-98, 2010

13. Kanaya H, Saito Y, Gama N, Konno W, Hirabayashi H, Haruna S: Intramuscular hemangioma of the masseter muscle with prominent formation of phleboliths: A case report. Auris Nasus 
Larynx 35(4):587-591, 2007.

14. Lee JK, Lim SC: Intamuscular hemangiomas of the mylohyoid and sternocleidomastoid muscle. Auris Nasus Larynx 32:323327, 2005

15. Lee SK, Kwon SY: Intramuscular cavernous hemangioma arising from masseter muscle: a diagnostic dilemma. Eur Radiol 17:854-857, 2007

16. Seccia A, Salgarello M: Treatment of angiomas with sclerosing injection of hydroxypolyethoxydodecan. Angiology 42:23-29, 1991.
17. Tang P, Hornicek FJ, Gebhardt MC, Cates J, Mankin HJ: Surgical treatment of hemangiomas of soft tissue. Clin Orthop Relat Res 399:205-210, 2002

18. Top H, Barcin E: Posttraumatic intramuscular hemangioma of the left temporal muscle. Eur J Plast Surg 27:210-212, 2004

19. Watson WL, McCarthy WD: Blood and lymph vessel tumors. A report of 1056 cases. Surg GynecolObstet 71:569-588, 1940

20. Wolf GT, Daniel F, Krause CJ, Kaufman RS: Intramuscular hemangiomas of the head and neck. Laryngoscope 95:210-213, 1985 